\title{
A topological reading of Ernesto Laclau
}

Pitagora: La regola sta al principio.

Eco: $M a$ trasporti questo atteggiamento nella vita sociale e politica. Cosa ne nasce? Una visione aristocratica e conservatrice. Non a caso Lei ha dovuto fuggire da Crotone, perché il partito democratico vedeva nella Sua scuola un centro di pensiero aristocratico e reazionario. Nella vostra fiducia nelle leggi eterne del mondo voi pitagorici non potevate comprendere la mutazione, [...] che la realtà nasce anche dal dolore, dalla lotta, che l'armonia è un punto d'arrivo, sempre provvisorio, ma guiai a considerarla un punto di partenza, definitivo.

Pitagora: Dunque non hai capito.

U. Eco, Pitagora ${ }^{1}$

\section{Introduction}

Ernesto Laclau asserts that the category of 'relation' is central to his analysis, and that this distinguishes his approach from other contemporary theories. In his own words, "Alain Badiou, for instance, sees set theory as the terrain of a fundamental ontology. Given the centrality to set theory of the notion of extensionality, however, the category of relation can, at best, play only a marginal role." ${ }^{2}$ The centrality to topology of the notion of relation ${ }^{3}$ suggests that a topological reading of Laclau may be fruitful. This work is an attempt to relate Laclausian categories and topology from the particular perspective of the theory of dynamical systems. Such theory has instability as its central category, but in order to study instability, dynamicists resort to topology. The categories of

1 Umberto Eco a Pitagora, Le interviste impossibili, Bompiani 1975, available at: http://www. giutor.com/doc/pitagora/eco-pita.html.

2 Ernesto Laclau, On Populist Reason, Verso, London and New York 2005, p. 68.

3 Jean Ladrière, El reto de la racionalidad, La ciencia y tecnologia frente a las culturas. Ediciones Sigueme, Salamanca/UNESCO, Paris 1978, p. 31.

* Centre National de la Recherche Scientifique (CNRS), France; (2) Institut Franco-Argentin d'Études sur le Climat et ses Impacts, UMI 3351 (CNRS-CONICET-UBA-IRD), Buenos Aires, Argentina 
relation and instability are therefore simultaneously central to the marriage between topology and dynamics.

The methods used to build a topological structure associated with a system's dynamics are developed in the so-called "topological program". ${ }^{4}$ This work constructs an analogical correspondence between concepts of the topological program and the main categories in Laclausian theory. The possibility of drawing a parallel between such disparate areas of thought relies on the fact that both approaches constitute efforts to describe structures resulting from an articulatory practice. The starting point is, in both cases, a series of disaggregate elements. A remark is necessary before we embark on this task. The analogy is not intended to reduce theoretico-political categories to a mathematical scheme, or to postulate the topological program as a Laclausian matheme. The aim is to outline a two-way relationship between the two conceptual fields that leaves neither of them unaffected. If the analogy unfolds a productive set of theoretical affinities, these may both lead to alternative formulations of some problems in political theory, and contribute to deconstructing the nature, conception, and uses of mathematical tools. ${ }^{5}$

This article is organised as follows. An introduction to the topological program is provided in Section 1. Section 2 is devoted to the premises of the analogy. Conceptual correspondences are presented in Section 3. The productivity of the analogy is discussed in Section 4.

\section{Section 1. Topology and Dynamical Systems}

A dynamical system is a system whose evolution is dictated by rules ${ }^{6}$ which, in the more general case and by their mathematical form, combine determinism

4 Robert Gilmore and Marc Lefranc, The Topology of Chaos: Alice in Stretch and Squeezeland, Wiley, New York 2002. The spelling "program" will be used herein for the sake of consistency.

5 Blaise Pascal and Henri Poincaré are some of the precursors in this effort.

6 The rule predicts the evolution of the system. More precisely, it predicts the evolution of certain variables that are assumed to characterise the system. A physical pendulum oscillating within a plane, for instance, is a system that can be described using two variables: position and velocity. In the example of the pendulum, the rule can be derived from consideration of the forces that act upon it. How many variables are needed to adequately describe a problem? This depends on the problem. Once the variables are chosen, how the problem is to be attacked is defined, and this limits what can be said about the system to what can be said 
with unpredictability. The theory of dynamical systems proposes a set of mathematical tools to study the phenomena governed by these kinds of rules (nonlinear rules). Given an evolution rule, initial values are chosen for the variables describing the system and the rule is used to compute the values of the variables that determine the subsequent states ${ }^{7}$ of the system. For the case of nonlinear rules, predictability becomes difficult for one reason: an astonishingly small difference in the initial value chosen for a variable makes the system evolve in a considerably different way. This is how unpredictability enters the scene without opposing determinism. A future state fails to be predicted not because there is no rule governing the system, but because the rule gives rise to radically different results for slightly different initial values. In order to avoid unpredictability, infinite precision is needed in the knowledge of the initial state and in the application of the rule. But this infinite precision is generally unavailable, not only in the case of experimental data, but also for numerical computations. A tiny difference that can make the system evolve in remarkably different manners is exponentially amplified by the mathematical nature of the rule that governs the system, hindering predictability in the long term. The error that is inherent to every measure or numerical computation brings "the contingent in the necessary." In the field of dynamical systems, this property is known as sensitivity to initial conditions. When data are generated with a nonlinear rule, the data present a seeming disorder. In other words, the rule governing the data is not transparent when the data are examined directly, but is concealed. This is why dynamicists talk about unveiling an underlying dynamics from data of this type. And this is why it is of interest to learn, for a time series of experimental data presenting this seeming disorder, if there exists - or not - an underlying deterministic dynamics. Notice that the finding of an evolution rule for such a system will entail an understanding of the mechanisms involved in the behaviour of the system. Scientific knowledge is not restricted to prediction.

about the variables. If the pendulum has a fixed length $z$ and moves within a plane, position and velocity are sufficient. Variables are also called state variables or dynamical variables.

7 A system is in a given state when the ensemble of variables that characterises it adopts a value. To correctly define the state of a pendulum with variable length, for instance, values should be assigned to three variables: position, velocity, and length. If the length cannot change, the length is again a parameter and the state of the system is defined by assigning values to two variables: position and velocity.

8 Ernesto Laclau and Chantal Mouffe, Hegemony and Socialist Strategy, Verso, London and New York 2001, p. 114. 
The topological program devises methods to unveil the type of organisation underlying a data series. The starting point is a time series associated with the phenomenon that one is attempting to understand. The rule is not known in advance, but it is tacitly supposed to exist. In other words, the system is supposed to be deterministic. The first step in the analysis consists in representing the data in a mathematical space, called phase space, in which time is implicit and in which each point stands for a state of the system. Notice that this space does not have a predetermined dimension. Its dimension depends on the number of significant variables in the problem. Representation in phase space ${ }^{9}$ does not focus on when exactly the system visits a certain state, but on whether there are privileged states to which trajectories tend to evolve, if there are sets of states that are forbidden, and so on. Representation in phase space breaks with the synchronic-diachronic dichotomy. ${ }^{10}$

When the system evolves from a starting point representing its initial state, it visits a sequence of states, leaving a trace in phase space called a trajectory, as shown in Fig. 1. A trajectory in phase space is a line that is read in the direction in which time evolves. This direction is indicated with an arrow. If the rule that governs a system is known, the rule can be used to compute trajectories in phase space. Trajectories that obey a certain rule do not circulate freely. Their route is dictated by the rule that governs the dynamical system.

9 Phase space is a geometrical space that enables a representation of the states of the dynamical system. Each point in phase space corresponds to a value of the variables that characterise the system, i.e. each point in phase space stands for a possible state of the system that is defined with those variables. It could also be called 'state space'. In the case of the pendulum, phase space can be constructed using two coordinate axes. One for the position of the pendulum $(x)$ and one for the velocity $(y)$. In this case, phase space has two dimensions just because two variables are enough to identify a state of the system. If we consider the length $z$ as a variable, we would need three coordinate axes $(x, y, z)$. The phase space dimension is determined by the number of variables that define a point (a state of the system) in phase space. Phase space therefore depends on the number of relevant variables of the problem. Let us stress that phase space is a mathematical space that has nothing to do with physical space.

10 Phase space provides neither a photograph of an ensemble of variables, nor a film of successively ordered events. If a system recurrently visits a zone in phase space at different times, a dense cloud of points will be found in this zone. It is a mathematical space that focuses on the representation of dynamical states. 


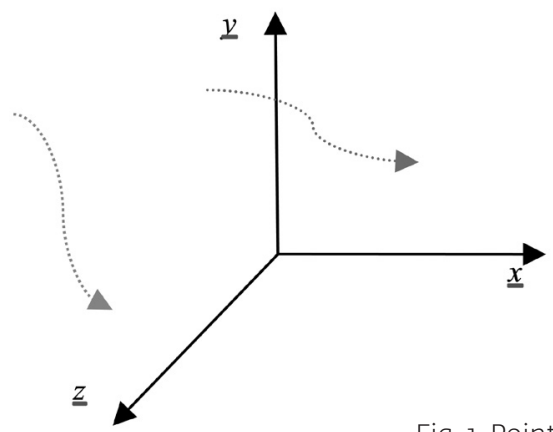

Fig. 1. Points forming trajectories in phase space for sequences of values for variables $x(t), y(t), z(t)$ as they evolve in time $(t)$ for two different initial states.

In order to illustrate what a privileged state in phase space looks like, let us imagine a trajectory ending up in a point. This means that the system has visited a certain number of states and reached a state from which it no longer moves. This state is called a stationary state. If this point attracts trajectories to it, this point is a privileged point in the system, to which the system tends to evolve. The point is said to be an attractor of the system. An attracting point absorbs trajectories in phase space (Fig. 2a). In the same manner, one can imagine repellers, i.e. states in phase space from which the system escapes (Fig. 2b). These privileged states structure trajectories in phase space. Sometimes there are groups of states that play this role. A limit cycle, for instance, is an attracting set of points in the form of a closed curve. Systems that converge to a limit cycle are systems that attain a stable and periodic (cyclic) behaviour (Fig. 2c).

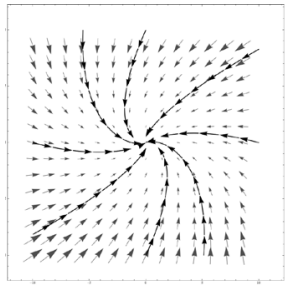

(a)

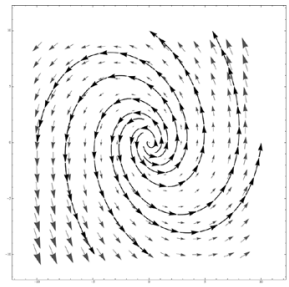

(b)

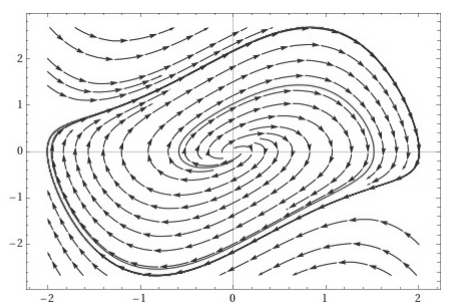

(c)

Fig. 2. (a) attracting fixed point; (b) repelling fixed point; (c) attracting limit cycle. 
When a trajectory falls into an attractor, it is clear that even if the system is slightly pushed away from this state it tends to fall back into it. An attractor attracts the points that lie in a certain basin of attraction in phase space. Why is this description in terms of attractors useful? Dynamical systems in the physical world tend to be dissipative, i.e. change tends to cease unless there is a force that is permanently acting upon the system. In those systems in which an acting force and dissipation coexist, the system tends to evolve (after a transient) to some kind of typical behaviour that is illustrated by attractors in phase space. Of course, several attractors can coexist in phase space for a given system. The evolution of trajectories is in this case conditioned by the basin of attraction in which trajectories are born.

Let us suppose that there is only one variable available for measurement. The starting point of the analysis is a time series. This variable may not exhaust the number of variables that are necessary to define a particular state of the system. In such case, there exist mathematical criteria for deciding the minimum dimension needed to host the data, and there are recipes to build supplementary series from the data. Once the time series have been embedded in phase space, clouds of points or states are obtained.

Let us consider the example in Fig. 3. The cloud of points comes from a representation of the pressure values recorded by a microphone when a speaker pronounces the sound /a/ in the first vowel of the Spanish word 'casa'. Two supplementary series are generated to achieve an adequate representation in phase space, which is three dimensional in this case. The result is shown in Fig. $3 \mathrm{~b}$.

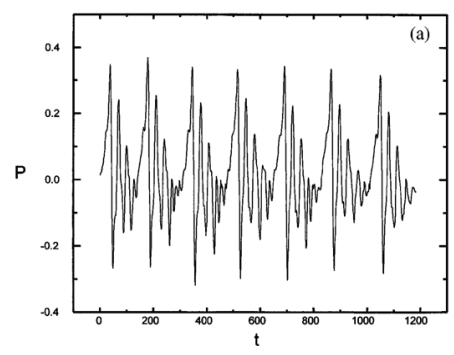

(a)

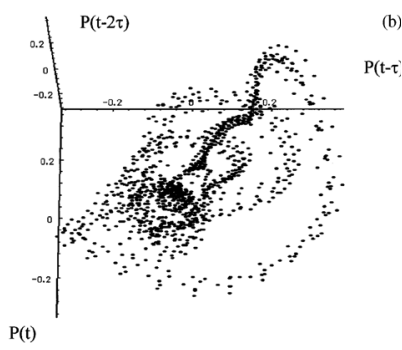

(b)

Fig. 3. Example of a time series (a), embedded in phase space (b). 
The next step is to construct, from this cloud of points, a sort of skeleton or model kit that articulates the points, according to the manner in which they are organised. This 'manner' is not geometrical but topological: what defines the skeleton is not the location of the points in the cloud, but the structure organising the cloud of points. Such a skeleton has been named a template or branched manifold. The term branched manifold makes reference to the fact that the skeleton usually takes the form of a structure of connecting branches. This structure can have isolated pieces that are not connected to the others; it can also be of one piece (one connected component). Each piece can contain branches, disks, holes, torsions, etc. The template is thus the graphical representation of a series of structural properties, which are topological in nature.

But how can this skeleton be reconstructed from the disperse points representing the states visited by the system? One of the methods to construct the skeleton $^{11}$ uses a procedure that is quite straightforward. ${ }^{12}$ A sort of mask is constructed covering the points displayed in phase space. This mask is built piece by piece, as shown in Fig. 4. The first step in the method consists in gathering points within cells or patches that will gradually cover the cloud of points. Points are grouped in cells provided there is a certain 'local affinity' or equivalence, which is defined in geometrical terms. ${ }^{13}$ Scattered points distributed in the cloud are arbitrarily chosen as 'nodal points' (Fig. 4a), which will nucleate nearby points around them. How many points should be nucleated around a nodal point to form a cell? As many as the affinity criterion allows to gather. If

${ }_{11}$ Denisse Sciamarella and G. B. Mindlin, “Topological Structure of Chaotic Flows from Human Speech Data”, Physical Review Letters 82 (7/1999).

12 The method of branched manifold analysis through homologies is presented in Denisse Sciamarella and G. B. Mindlin, "Unveiling the Topological Structure of Chaotic Flows from Data”, Physical Review E 64 (3/2001). Before this work, alternative approaches were based on knot theory. These were not applied to the mask (the manifold) where the data lie, but to a prior reconstruction of orbits approximating the trajectories described by the data. The reconstruction of orbits is difficult if the time series is noisy, as in Fig. 3b. Approaches based on knot theory have the additional drawback of being limited to cases in which the dimension of phase space is lower than or equal to three, since knots fall apart in higher dimensions.

${ }_{13}$ In the method, the affinity criterion is expressed geometrically in the following manner. The group of points in a cell must be a good approximation of a hyperplane of dimension $d$ in a space of dimension $n$. 
the number of points per cell around the nodal points is sufficiently large, cells start overlapping, as sketched in Fig. 4b. This favours concatenation between cells. The points linking cells are called vertices. Each cell can now be labelled using a sequence of vertices, numbered in Fig. 4c. Notice that cells are polygons that do not necessarily adopt the form of the points that are gathered to constitute a given cell, but this does not matter, because even if the criterion applied to the points to construct the cells is geometrical, a given cell is not a geometrical approximation of the points, but a topological approximation of the structure in which the points are inscribed. This point will be central to the analogy: cells represent groups of points without coinciding with the points, or with the frontier points, or with the trajectories that the points are supposed to make up.

Together, the cells constitute a cell complex. The complex inherits the structure of the embedded data regardless of the individual trajectories, which are not even reconstructed. The equivalential bond between points operates locally, ignoring the trajectory to which the point belongs, and by virtue of a criterion that is ultimately arbitrary: the laxer the criterion, the greater the affinity between the points and the higher the chances of conforming to a one-component complex. This 'loss of memory' of the individual trajectories is not destructive. The cell is transparent and makes up a parallel structure, which is constructed to express the logic of an organisation related to a particular dynamics. It is clear that if the criterion is too rigid or too strict it will hinder the construction of the complex. Nodal points are also arbitrary: a different collection of nodal points may lead to a different distribution of cells.

The model kit resists this variability because the aim is to reconstruct the underlying topology. Once the vertices are labelled with numbers, the labels can be used to denote paths along the complex. One of the paths of Fig. 4c, for instance, is obtained with the cyclic sequence: $1,2,6,9,19,12,5,1 .{ }^{14} \mathrm{~A}$ chain is any sequence of elements of the cell complex. In this case, the cell complex has o-cells (vertices), 1-cells (lines), and 2-cells (polygons). Sequential chains are important

14 Vertices are also called o-cells, and chains of vertices are called 1-cells. Chains can also be formed with sequences of cells, e.g. $\langle\langle 2,3,7,6\rangle,\langle 9,8,7,6\rangle,\langle 10,8,9,11\rangle\rangle$, called 2-chains. The number in o-cell or 1-chain denotes the dimension of the element of the complex. A o-cell is a point, while a 1-cell or 1-chain is respectively a segment or sequence of consecutive segments. A 2-cell or 2-chain is a polygon or sequence of concatenated polygons, respectively. 
because the organisation of the complex is provided by the manner in which cells are connected to other cells.

In order to determine the topological properties of the complex, another equivalential relation is needed. This equivalential relation is called homology. It allows the determination of in how many non-redundant ways the complex can be visited, so that the holes, the number of bands or handles, and the number of enclosed cavities (if there are any) come out. Two paths are equivalent when one can be continuously deformed into the other. Unlike the criterion operating to constitute connected cells in a complex, the criterion for deciding if two chains of the complex can be continuously deformed into each other is neither arbitrary nor geometrical: it is equivalential and topological. The equivalential logic that enables the construction of the cell complex, with its arbitrary character,

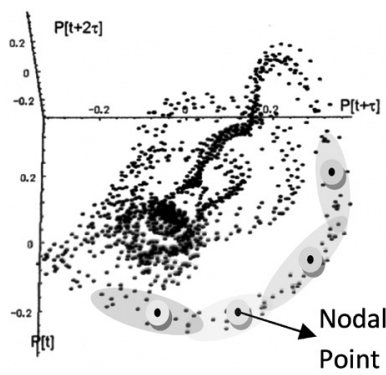

(a)

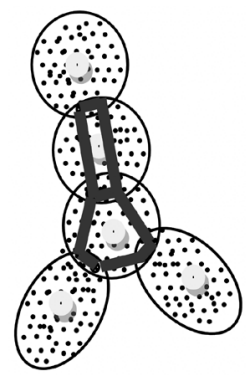

(b)

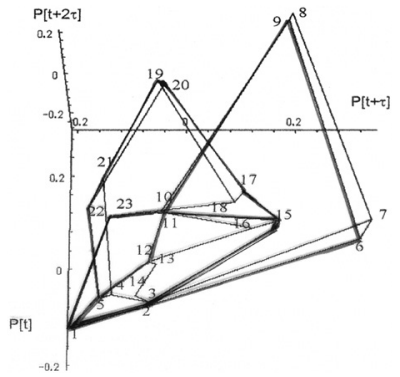

(c)

Fig. 4. From the cloud of points to the cell complex.

differs from the equivalential logic that unveils the organisation of chains. It is the combination of both that finally leads to the articulatory skeleton organising the plurality of sites in phase space.

Once the cell complex is obtained, it can be characterised in a "layered" way by answering these questions: How many connected components does it have? How many inequivalent loops can we draw on it? Does it enclose cavities? The answers, which can be calculated using algebraic topology, are the topological 
properties of the cell complex. These properties lead to the template through the computation of the homology groups..$^{15}$

Homology groups code structural information. ${ }^{16}$ If we go back to the example of the acoustical pressure, homology groups yield the structure that articulates the data of our example. These paths are the three inequivalent loops that articulate this structure, and that can be used to design the template. If we label the complex in Fig. $4 \mathrm{C}$ as complex $\mathrm{K}$, the result can be written as follows: $\mathrm{H}_{\mathrm{o}}(\mathrm{K})=[[<1>]]$ $\sim \mathrm{Z}^{1}$ (we have a 1-piece structure); $\mathrm{H}_{1}(\mathrm{~K})=\left[\left[\mathrm{L}_{1}, \mathrm{~L}_{2}, \mathrm{~L}_{3}\right]\right] \sim \mathrm{Z}^{3}$ (with three inequivalent loops or branches, the three cycles in Fig. $4 \mathrm{C}$ ) and $\mathrm{H}_{2}(\mathrm{~K}) \sim \emptyset$ (no enclosed cavities). It can also be noted that there is a torsion in cycle $L_{2}(T(K)=\{<2,3>\})$. The three inequivalent cycles make up two bands or branches, which reconnect

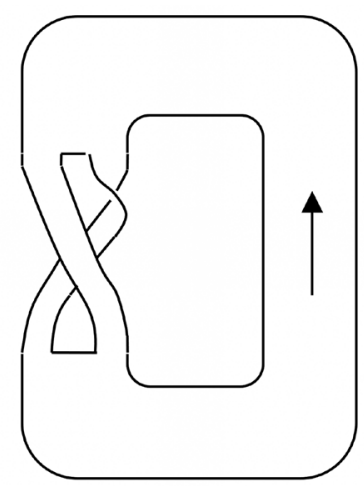

Fig. 5. Template corresponding to the organisation of the data in Fig. 4C.

15 Homology groups are a series of layered sets that condense the information on how the plurality of points is articulated in the topological structure. The set at level o (usually labelled $\mathrm{H}_{\mathrm{o}}$ ), which condenses information on o-cells, indicates the number of connected components of the structure. The set at level 1 (labelled $\mathrm{H}_{1}$ ) contains the information on the number of non-redundant loops that can be constructed using 1-cells. The set at level 2 (labelled $\mathrm{H}_{2}$ ) indicates the number of enclosed cavities through the non-redundant paths that can be constructed with 2-cells, or polygons. The example of Fig. $4 \mathrm{c}$ makes us stop at level 2 because the cell-complex does not have 3-cells or polyhedra (the manifold is locally bidimensional).

${ }_{16}$ Here are a few examples of different topological structures in terms of homology groups. The cylinder has one component in $\mathrm{H}_{0}$, one element in $\mathrm{H}_{1}$, and o elements in $\mathrm{H}_{2}$. The torus has one component (one connected piece) in $\mathrm{H}_{\mathrm{o}}$, two components (two inequivalent loops) in $\mathrm{H}_{1}$, and 1 component (one enclosed cavity) in $\mathrm{H}_{2}$. 
in a central disk, with a torsion in one of the two branches. The corresponding branched manifold or template is shown in Fig. 5.

The reason why topological properties are interesting is that data associated with the same rule lead to the same topological structure. The template therefore shows how the data are organised in phase space under the action of the underlying deterministic rule, outlining an "ordre dans le chaos." classifies manifolds ${ }^{18}$ in a qualitative manner, ignoring deformations that do not introduce (or remove) holes or cuts. ${ }^{19}$ What does topology identify in an object? Topology identifies disconnected components, holes, enclosed cavities, and so on. It does not identify sizes, distances, or continuous deformations.

Going back to the example in Fig. 3, suppose that the speaker is asked to repeat the word several times. If the rule governing the pressure time series does not change as the speaker repeats the word, the geometrical distribution of the cloud of points may change, but the topological structure organising the skeleton should still be that of Fig. 5. The template structuring the data is said to be an invariant, ${ }^{20}$ a topological invariant. Dynamical systems can therefore be classified according to their topological structure, so that unveiling the topological structure amounts to unveiling the governing rule. The topological program is a guide leading from the data to the rule.

A few remarks are necessary. First of all, the mathematical procedure for obtaining the template from the data is not unique: several methods have been proposed. The method we have outlined above has the particularity of applying the concept of homologies to the 'mask' in which the data lie. Homology is

${ }_{17}$ Pierre Bergé, Yves Pomeau and Christian Vidal, Lordre dans le chaos: vers une approche déterministe de la turbulence, Hermann, Paris 1988.

18 A manifold is a mathematical space that on a small enough scale resembles the Euclidean space of a specific dimension, called the dimension of the manifold. For instance, the surface of a sphere is a manifold of dimension 2, because on a small enough scale it resembles a plane.

19 Topologically, a sphere and a plane are different objects. But topology would not distinguish a slightly deformed sphere from one that was not deformed. A sphere and a cylinder, for instance, are topologically different, because it is impossible to deform one into the other, unless two lids are cut from the sphere or glued to the cylinder.

20 The concept of invariance has been extremely fertile in physics. A classic example of an invariance principle is the conservation of energy. 
an equivalence relation between the elements that constitute the cell complex. Secondly, the topological properties obtained in the application of the topological program are data dependent. This means, on the one hand, that results are not always reliable: they can even be deceptive if the data series is too short, for instance, or if it is too noisy. Thirdly, the rule that is attained through the topological strategy does not exhaust the possibilities regarding the behaviour of the system. The topological program describes a dynamics that is encrypted in the data under analysis, but it does not encompass the totality of mechanisms governing the system from a meagre series of data. More precisely, the mechanisms that will be unveiled by the topological strategy are those that are contained in the data. If the data series is enlarged, the template may be altered. Topological changes in the template may have different origins: a variation in the parameters ${ }^{21}$ involved in the rule generating the data, a change in the mathematical form of the rule, or the necessity to add variables hitherto disregarded. In short, the template is just the manner in which the data under analysis are articulated in a space that has been conveniently defined to host the dynamics of the data. The template condenses a relational necessity ${ }^{22}$ which differs from the natural necessity of the system that it partially represents. Topology provides an invariant expressing a relational necessity that, in the case of deterministic systems, can be used as a guide towards the natural necessity expressed by the rule.

\section{Section 2. The premises of the analogy}

Let us examine on what grounds the concepts of topology applied to dynamical systems can work as analogues of the main Laclausian categories.

First of all, Laclau and Mouffe maintain that the social order cannot be understood as an underlying principle. ${ }^{23}$ This would represent an obstacle if our aim was to apply the topological program to data of socio-political origin, because

${ }^{21}$ Parameters can be thought of as variables that are supposed to remain constant for the system. Parametric studies typically show that the template remains the same for certain ranges of parameter values. It is in the jump from one range to another that a change in parameter values may create or delete branches in the template.

22 Ernesto Laclau and Chantal Mouffe, Hegemony and Socialist Strategy, Verso, London and New York 2001, p. 154.

${ }_{23}$ Ibid., p. 96. 
the goal of the program is to unveil an underlying rule. Nothing prevents, in fact, the application of the topological program to socio-political data, but undertaking this task implies either that there is or that there can be an underlying principle in the inspected data. Moreover, the choice of the time series for the analysis (e.g. the evolution of the human poverty index developed by the United Nations in a certain region and time interval) pre-identifies the type of underlying principle that is being looked for. But this is not what is being proposed in this work, which is analogical and not mathematical. The connection between the topological structure and the mathematical rule is only the last step of a method that otherwise deals with the problem of converting a cloud of states representing a dynamics into a skeleton. The last step can be taken if the system is deterministic, and is successful as long as the elements excluded from the analysis remain dynamically irrelevant. In Laclausian terms, the last step is suturing, ${ }^{24}$ inasmuch as it transforms a fixation that is partial and open into a fixation that is closed and complete. It is a reasonable step for dynamicists in search of models, but an undesired leap in post-fundamentalist political theory. It can therefore be advanced that the analogy will take up conceptual elements of the method leading from the disperse data to the topological structure, leaving out the leap from topology to determinism, in the assumption that there is not, and there cannot be, an underlying principle of the social. Assuming that there is an underlying principle of the social is condescending to the metamorphosis of a hypothesis into a prescribing principle. In order to illustrate the dangers of establishing a principle of the socio-political through mathematical models inspired by the theory of dynamical systems, the reader is referred to other texts. ${ }^{25}$ In short, the topological program is not invoked as an analytical tool of data of socio-political origin. Nor is it suggested that the logic of the political should be, for some mysterious reason, topological in nature. The analogy does not attempt to reduce the gap separating a social problem from a deterministic problem.

24 The concept of suture is taken from psychoanalysis. Hegemonic practices are suturing in as much as they attempt to complete the incompletable. According to Laclau and Mouffe, such a closure of the social is impossible.

25 For a case study on the concept of model in politics, see Amilcar O. Herrera, et al. ¿Catástrofe o nueva sociedad? Modelo Mundial Latinoamericano. Centro Internacional de investigaciones para el Desarrollo, Agencia Nacional de Investigación y Desarrollo, Santiago de Chile 2004. 
Secondly, the analogy is possible as long as we are ready to sacrifice a number of characteristics that are attributed by default to the naturalist paradigm. Laclau and Mouffe maintain that the type of objectivity constructed by the discourse of the natural sciences sets limits that exclude the notions of metaphor, analogy, metonymy, or contradiction. The naturalist perspective is therefore considered to be incompatible with the theoretical development that follows. But is this so? Are all the toolboxes, in the Foucaultian sense of the expression, developed by natural scientists or mathematicians unproductive when used in an unprescribed manner? This proposal is based on the belief that unprescribed uses are not always infertile, and that thought is not ordered in sealed compartments. Blaise Pascal, who promoted the mixture of heterogeneous sources, asserted that there can be harmony where we do not expect to find it, and there can also be fusion problems in the homogeneous. ${ }^{26}$ Other authors have raised and analysed questions concerning the contingency of the a priori, the problem of language mediation in the naturalist paradigm, of partialness in formal languages, or of mathematics and metaphor. ${ }^{27}$ Using a 'language ${ }^{28}$ is a practice that can be compared to accepting the perspective of a picture. Taking concepts of two 'languages' and mixing them, or setting them face-to-face, as two mirrors, can produce perspective effects that may appear as strange, but that are not necessarily unfruitful, especially if we believe, with Pascal, in the possibility of harmony between irreducible perspectives.

Thirdly, let us mention a few points that seem to favour the formulation of the analogy. One of these points is the defence put up by Laclau as to the compatibility of a partial order with the absence of an ultimate principle. ${ }^{29}$ The topological program is, after all, a strategy for searching for an organisation that is conditioned by a series of choices relative to the representation of a system in phase space. This organisation can be altered, as mentioned before, in a series of manners, which have been typified. Another point has to do with the character of the program, which is not topological tout court. It is already a marriage

26 Maurice Caveing and Évetyne Barbin, "Les Philosophes et les Mathématiques”, Raison présente 123 (1997).

27 Vladimir Tasić, Mathematics and the Roots of Postmodern Thought, Oxford University Press, Oxford 2001.

${ }_{28}$ Whether mathematics is or is not a language is a controversial issue. See, for instance Jean Petitot-Cocorda, Morphogenèse du Sens, PUF, Paris 1985, p. 19.

29 Laclau and Mouffe, Hegemony and Socialist Strategy, p. 114. 
between topology and dynamics. The topological structure is a structure constructed not in physical space, but in phase space, and as a consequence it is associated with the deployment of a dynamical behaviour. The categories in Laclau's theory aim at a description of fixations that are representative of certain processes. Last but not least, there is a series of terminological coincidences between Laclausian theory and the topological program that invite this analogical exercise. The concepts of equivalence, chain, and nodal point are central to the construction of the template through homologies, and they are also central to the Laclausian conception of a popular identity. The concept of regularity in dispersion ${ }^{30}$ may redirect to the mechanisms leading to the emergence of topological invariants in nonlinear systems, in which regularity and dispersion coexist. Dispersion is present through the sensitivity to the initial conditions, which makes nearby initial conditions diverge after a certain time. Regularity is present by a certain recurrence in phase space, where motion is eventually bounded. These two 'contradictory' mechanisms - dispersion and contraction force nearby trajectories reaching maximum separation to begin to approach each other again..$^{31}$ The fundamental idea of the topological program is that the regularity and dispersion processes, applied in a non-periodic manner, provide a sort of partial fixation. ${ }^{32}$ Topology provides the embodiment of this partial fixation. Fixation is partial because the topology invariance can be subverted by an exterior ${ }^{33}$ constituted by all that is left out of the analysis: the disregarded variables, the mechanisms that are not expressed by the data series, etc.

Fourthly, let us make a few remarks concerning the usage of topological concepts in non-mathematical texts. If Laclausian categories are constructed in view of some of the philosophical ideas introduced by Lacan, a legitimate question is whether the 'affinity' between topology and Laclausian categories is somehow 'inherited' through Lacan, who does appeal to topology. Our analogy

$30 \quad$ Ibid., p. 106.

${ }^{31}$ These opposite tendencies are reconciled by operating in different directions, and this explains why there is no chaos (no complex, long-term unpredictable behaviour) in two-dimensional phase space. The third dimension is necessary for divergent trajectories to be squeezed back without self-intersecting. See, for instance, Pierre Bergé, Yves Pomeau and Moniques Dubois-Gance, Des rythmes au chaos, Editions Odile Jacob, Paris 1994, p. 118, or Gilmore Robert and Marc Lefranc, The Topology of Chaos: Alice in Stretch and Squeezeland, p. 127.

32 Laclau and Mouffe, Hegemony and Socialist Strategy, p. 113.

33 Ibid., p. 111. 
is not based on Lacanian references to topology. If it were, the analogy would be conditioned by pre-established connections, or by the kind of usage that Lacan makes of topology. ${ }^{34}$ Lacan borrows from topology a series of specific objects, such as the torus, the Möbius strip, and the Klein bottle. These objects are particular topological structures, and, as such, they are examples of the many possible results of the application of the topological program. But the parallel drawn in this article is not rooted on the topological objects themselves, but on the conceptual elements leading to the construction of a topological organisation. It is on the topological program as a method (and not on its possible results) that the analogy is built. Therefore, if there is a Lacanian influence underlying this affinity, it can probably be traced back to more general questions. The use of topology in Lacan may be seen as motivated by the inadequacy of the opposition interior-exterior to pose certain problems. The irresolvable interiority/exteriority tension is central to Laclau's arguments, and it can also be seen as essential to the preservation/subversion of topological invariance in phase space. Let us recall that the topological structure is always threatened by an exterior. 35

Before we move on to the terms of the analogy, a last comment may be pertinent as to the mathematical character of the topological program. Being mathematical, the program does not restrict its application to a particular kind of data: the origin of the data need not be specified for the program to be applied. But if this is so, what exactly delimits the context, the original terrain, of the topological program? The terrain is delimited by the operating rules underlying the practice of the program. Some of these rules are explicit: for instance, the program applies to deterministic systems. ${ }^{36}$ To allow for the analogy, the topologi-

34 Lacan appeals to mathematics as a formal system of writing capable of transmitting integrally, without remainder, a piece of psychoanalytic knowledge [un savoir psychoanalytique]. Alain Badiou, another author that takes up some of Lacan's concepts, and whose works contain mathematical inscriptions, does not operate in Lacan's way. He "seeks to capture the power of mathematics for the sake of a conceptual development.” Alain Badiou, The Concept of Model: An Introduction to the Materialist Epistemology of Mathematics, ed. and trans. Zachary Fraser and Tzuchien Tho, re.press, Melbourne 2007, p. xi.

35 In this sense, it is a mathematical object that could be described, to borrow Rorty's expression, as radically contingent. Ernesto Laclau, Emancipation(s), Verso, London and New York 2007, p. 118.

36 The transparency of tacitly operating rules in mathematics has been analysed by several authors. It has been held that, if formal language supresses the ambiguity of ordinary lan- 
cal program must be taken out of context at least in this sense. This contextual displacement can be compared to the change of focus carried out by Freud from the mystery of the form: "the 'secret' to be unveiled through analysis is not the content hidden by the form (the form of commodities, the form of dreams) but, on the contrary, the 'secret' of this form itself". ${ }^{37}$

Similarly, the analogy is focused not on the mystery behind the form of the data in phase space (the governing rule), but on the mystery of this form and its construction, on the articulatory mechanisms of a structure representing an open dynamics. ${ }^{38}$ The contextual displacement can be virtuous if the interest in topological invariants is exclusively guided by cogitation on the concepts of articulation and instability on the same relational playground. A certain distortion of the original tools is associated with the contextual displacement, since these tools are not used to calculate or to unveil an unknown, but to reflect upon certain politico-philosophical categories. Such a distortion will affect, for instance, the status of some of the elements of the topological program. In the original terrain, the template is a concept with a quasi-ontological status that stands independently of the method designed to unveil it, while the concepts of cell, chain, nodal point, equivalence, and homology are concepts with a methodological status. This distinction will not survive once the contextual displacement is operated. Cell, chain, nodal point, equivalence, homology, and template will have the status of conceptual tools allowing for an arrangement [agencement] $]^{39}$ of Laclausian categories.

guage, this is achieved at the price of preserving a particular kind of ambiguity: generality. Henri Poincaré asserted that we inevitably operate with certain mathematical prejudices, and that many notions (and the notion of identity is an example) are in fact pre-logical (they are motivated but not well-founded). These beliefs underlie every rational practice and constitute its unstated condition of possibility. Slavoj Žižek, The Sublime Object of Ideology, Verso, London and New York 2006, p. 1.

Open is used in the sense introduced by H. Poincaré. Objects that are impredicatively defined (invoking the reference to a totality) are never immutable or fixed. They are open because their identity is not present in them but maintained or supported by a nominative operation.

39 This expression is borrowed from Alain Badiou, who asserts that the effects of the philosophical text owe their force and duration to the mere arrangement [agencement] of concepts. Alain Badiou, The Concept of Model, p. xi. 


\section{Section 3. Analogical correspondences}

\section{Discourse}

The category of discourse is conceived by Laclau as a complex of elements in which relations play a decisive role..$^{40}$ The fact that objectivity and relation are synonymous is a principle that may also apply to phase space. Phase space is a field of representation that functions as an open relational complex between a number of state variables that are defined as pertinent. It fixes, at least provisionally, what will and will not be spoken about. The relations that cannot be represented in phase space are a result of an exclusion. The exclusion has practical reasons relative to the type of description that is attempted. Objects in phase space are therefore "threatened" by all that has been excluded, in an exclusion that is constitutive. Phase space is the primary terrain for the constitution of objects, and it is therefore the analogue of Laclau's "precarious totalising horizon," the "failed totality" necessary to start speaking about something.

Discourse is described by Laclau as being "split between the particularity which it still is and the more universal signification of which it is the bearer." Phase space can also be thought of in these terms, and these terms lead to the concept of hegemony. Hegemony is defined as the operation by which a particularity bears a totalising signification. Borrowing this definition, we could say that we talk about phase space by virtue of a hegemonical operation.

The term moment is used to refer to the differential positions insofar as they appear articulated within a discourse..$^{41}$ These definitions let us establish two initial conceptual pairs for our table of correspondences: the first one is the pair discourse-phase space and the second one is moment-state.

40 "Discourse is the primary terrain of the constitution of objectivity as such. By discourse, as I have attempted to make clear several times, I do not mean something that is essentially restricted to the areas of speech and writing, but any complex of elements in which relations play the constitutive role. This means that elements do not pre-exist the relational complex but are constituted through it. Thus 'relation' and 'objectivity' are synonymous.” Ernesto Laclau, On Populist Reason, Verso, London and New York 2005.

${ }_{41}$ Laclau and Mouffe, Hegemony and Socialist Strategy, p. 105. 


\section{Demand}

The minimal unit of analysis in Laclau's theory is the socio-political demand ${ }^{42}$ and the scope of his theory is aimed at consideration of how demands are articulated. In our parallel, let us tentatively associate demand and trajectory. A trajectory in phase space is just the path defined by a series of sequential states. The construction of topological objects in phase space is meaningful insofar as the topological object holds bundles of trajectories in phase space that are articulated together. The trajectory can therefore be seen as the minimal unit of analysis for topological organisation. Cross-relating the terms of the analogy, a demand will be born under the form of a trajectory that initiates a path in phase space.

What happens to a demand when it is satisfied? "A fulfilled demand ceases to be a demand." $\$ 3$ If the pair demand-trajectory is kept, we should be able to decide when a trajectory ceases to be a trajectory. If there exists a point in phase space that attracts trajectories that are born in different initial points covering a region in phase space, this point ends up absorbing the trajectory. This absorption makes the trajectory disappear. What happens to a system whose evolution is described by a trajectory that is absorbed by a point? It reaches a stationary state. We can imagine a group of initial states in phase space. After a transient, there is a state (a point) that is chosen as the definite dwelling. This point is called an attractor. But attractors are not always points. They can also make up closed curves in phase space. In such cases, the stationary state achieved by the system is not a single state but a closed sequence of states.

To proceed with the analogy, let us take a step further and associate the achievement of a stationary state with the satisfaction of a demand. The transient walk of the trajectory before it is absorbed by an attractor is the analogue of the time interval during which a demand is still only a request. "If the demand is satisfied, that is the end of the matter." 44 Otherwise, the trajectories persist in phase space without being absorbed. This persistence turns demands into claims and trajectories into recurrent trajectories, i.e. the trajectories associated with this recurrence and with the impossibility of reaching a stationary state function as the analogue of the unsatisfied demands. Let us consider a system for which all

\footnotetext{
Laclau, On Populist Reason, p. 224.

Ibid., p. 127.

Ibid., p. 73.
} 
trajectories find, after a certain transient, their attractor. An example of such a system could present several point attractors distributed in space, without barriers impeding the encounter between the trajectory and the dwelling that hosts it. This situation is the analogue of a society in which "any social need should be met differentially; and there would be no basis for creating an internal frontier." ${ }^{25}$ But what happens if an attractor transforms into a repeller, thus separating a certain group of demands from their satisfaction? This inaugurates clearings or voids, delimiting frontiers in phase space. Why? Not because trajectories cannot transit these regions, but because after a certain transient, none of them will survive in them. These voids delimit regions in phase space and define borderlines or frontiers. Internal frontiers are the first of a series of necessary preconditions for the emergence of populism.

The theory of dynamical systems provides tools to describe bundles of trajectories distributed in surfaces with branches and holes, trajectories that never become stationary, even if they never abandon a bounded region of phase space. This region that attracts trajectories without making them stationary is called a strange attractor..$^{46}$ It is an attractor because it is a set towards which trajectories evolve over time, but it is 'strange' because trajectories evolve in a recurrent but non-periodic manner, without ever repeating themselves.

The most famous example of a strange attractor is perhaps the Lorenz attractor. ${ }^{47}$ The rule that governs the Lorenz system is a nonlinear rule with three state

$45 \quad$ Ibid., p. 78.

46 These attractors are more complex than a point, a surface, or a volume in phase. Their particular feature is that they form geometrical objects with a non-integer dimension. A point has dimension o, a curve has dimension 1, a surface has dimension 2, but a strange attractor is a geometrical object that is, for instance, more than a curve but less than a surface.

47 The rule that governs the Lorenz system is a rule that can be derived from a simplified version of the laws for natural convection in the atmosphere. The Lorenz attractor is a paradigmatic example of a system in which the capacity of prediction is hindered. This is illustrated with the time series of the plot below.

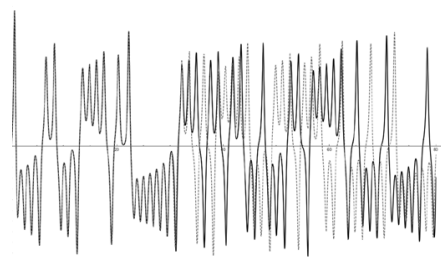




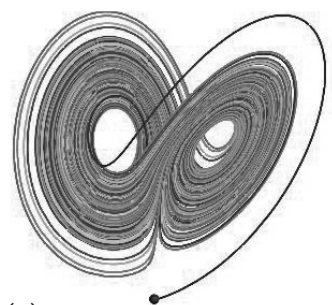

(a)

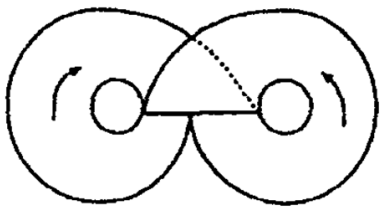

(b)

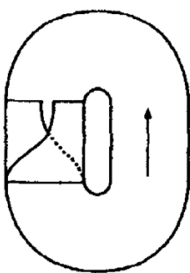

(c)

Fig. 6. (a) Trajectories in phase space generated with the Lorenz system. (b-c) Two alternative but equivalent forms of representing the model kit of the strip within which trajectories circulate.

variables: phase space is therefore three dimensional. Trajectories computed with this rule generate an object that is shown in Fig. 6a, with trajectories circulating in a sort of ribbon or strip, making up a locally bidimensional manifold. The strip circumscribes the trajectories but does not coincide with them. It is a sort of holder or container, which may be represented graphically with the mod-

The plot shows two-time series for one of the three state variables of the Lorenz system. These time series are obtained using the Lorenz rule. Both time series start at time zero, with $x$ values that are almost identical. The difference between both $x$ values amounts to 0.000001. During the first time period (which lasts about 15 seconds), the evolution of the state variable $x$ coincides for both time series and we can only see a single red curve. This poses no problem for prediction. But for a certain time the series that were coincident become drastically different, as if they had nothing in common. A prediction beyond the first 15 seconds with this rule will therefore fail. The rule is known but the rule does not suffice to predict the long-term behaviour of the system, due to sensitivity to the initial conditions. This kind of behaviour, illustrated here by the Lorenz system, is called chaotic. There is no consensus on a formal definition of deterministic chaos, but the term 'chaotic' is used to refer to deterministic phenomena described in terms of variables that vary in a particularly irregular fashion in time and space. It is known that a chaotic behaviour is constructed through the action of two basic (and opposed) mechanisms in phase space: stretching and squeezing. Stretching is connected with the property of sensitivity to the initial conditions. Squeezing is associated with the fact that variables adopt values within a limited range of values in phase space. For instance, in meteorology, pressure and temperature are variables that adopt values within a certain range in the atmosphere. When both mechanisms are simultaneously present, diverging trajectories in phase space end up approaching each other in the long run. An imperfect regularity results from the combined action of these two opposed mechanisms of dispersion and contraction, producing the 'mille feuille' object of Fig. 6 a. 
el kit shown in Figs. 6b and 6c. Both model kits are topologically equivalent. The first version resembles the attractor in Fig. 6a, but the strip can be continuously deformed until it coincides with the second version. ${ }^{48}$ The model kit in either (b) or (c) is the template of the Lorenz system.

The first important point to retain from this example is that trajectories that coexist with a strange attractor are distributed in phase space, forming bundles that can, however, be confined or inscribed in the structure that articulates them. The second important point is that trajectories trapped in a strange attractor move in a disorderly manner that does not appear to be generated by a rule, even if this is the case. But the structure in which the trajectories move can be of help to unveil the rule, when it is not known in advance.

The trajectories coexisting with a strange attractor can be conceived as unstable orbits that never close upon themselves to form a periodic orbit. We can think of a strange attractor as a set that hosts trajectories without absorbing them. Why does it host them? Because there is an object in phase space with well-defined topological features that contains them. Why does it not absorb them? Because stationarity is never reached.

Laclau remarks that the lack of fulfilment of a demand can take the form of a downright rejection - as in the case of a repeller expelling a trajectory from its zone of influence - but it can also take the form of an unstable equilibrium between satisfaction and rejection. ${ }^{49}$ This intermediate situation between satisfaction and rejection is comparable to the particular situation encountered by a trajectory that coexists with a strange attractor. These trajectories change permanently but within certain limits, and these limits preserve a mathematical form that is well described by topology. In the field of dynamical systems, we say that there is topological invariance and we associate this invariance with the dynamical properties of the rule governing the system. The strange attractor maintains its topology unless the system suffers radical changes (through a parameter variation, through the incorporation of dynamical ingredients that were absent, or through the inclusion of previously excluded variables). Within

\footnotetext{
$4^{8}$ Recall that the transformation process of one version into the other only forbids discontinuous actions such as cutting or welding.

49 Ibid., p. 120.
} 
the apparent disorder of trajectories that never settle to a stationary state, there subsists an order of a higher level: the level of the topological structure organising the trajectories.

But how can we jump from the level at which there is disorder - the level of the trajectories - to the level at which there is order - the level of the template? By establishing an equivalential relation at the level of the trajectories. The establishment of equivalential relations between demands is the second precondition for the emergence of populism.

What kind of equivalential relations are introduced in the topological program? In the first place, a local equivalence is practiced on nearby states, enabling the grouping of stretches of trajectories that admit a local ${ }^{50}$ common inscription, provided certain criteria are met. ${ }^{11}$ What comes out of the establishment of this local equivalential relation? A number of organised cells representing stretches of grouped trajectories, constructed around nodal points. This local equivalence leads from the trajectories to the template.

The Laclausian equivalential chain between demands presents trends that are similar to the equivalential bond constituting a cell in the cell complex. The more extended the equivalential relation, the laxer the criterion that is applied to constitute it. The arbitrary nature of this criterion has an analogue in the emptiness of the signifier: ${ }^{22}$ the laxer the criterion, the emptier the signifier. Another trend that admits an analogical translation is the ontological function overriding the ontic content..$^{53}$ The fact that nearby trajectories have nothing in common when they are followed out of the region of interest does not interfere with the inscription of their common stretch around a nodal point to form a cell. The ontological need to be unified under a signifier (or to be grouped under a criterion having an arbitrary common reference) is stronger than the ontic difference between demands (or mutually diverging trajectories). The method

50 The equivalence between the states in the cell is local because their continuation out of the cell can be completely different, but this does not matter.

${ }^{51}$ This local inscription can be seen as the analogue of a "levelling instinct", Ibid., p. 102.

52 "A signifier like 'workers', for instance, can, in certain discursive configurations, exhaust itself in a particularistic, sectional meaning; while in other discourses - the Peronist would be an example - it can become the name par excellence of the 'people'." Ibid., p. 87.

53 Ibid., p. 87. 
that we are describing does not even attempt a reconstruction of the trajectories from the disperse points corresponding to the available data. And this is not attempted because the identification of the trajectories does not matter to the reconstruction of the topological structure. The focus is on how disperse states are articulated in phase space, independently of the trajectories to which these states might belong.

The demands that enter into an equivalential relation with other demands are called popular demands. The construction of the cell complex in the topological program provides an image of the Laclausian equivalential process. Popular demands have their analogue in trajectories admitting an inscription within a cell complex. The analogy is even respectful of the fact that "equivalence does not attempt to eliminate differences. ${ }^{54}$ The cell does not replace the trajectories, it does not coincide with them, nor does it make the trajectories coalesce into a unique trajectory; the cell is an object that can be imagined as overlying or supporting different trajectories when certain criteria are met.

The topological program admits a second type of equivalential relation. It is an equivalence relation that operates upon the cell chains that can be defined along the cell complex, i.e. at the level of the structure supporting the initially disperse states and contributing to the determination of the topological properties of such structure. This equivalence relation between chains is termed homology and it leads to the recognition of the number of connected components, of inequivalent loops, of enclosed cavities, etc. This stage of the topological program can be seen as the third and last precondition for the emergence of populism: the unification of the various demands into a stable system of signification. ${ }^{55}$

\section{Institutionalism}

Let us turn back to the case in which phase space is populated with non-strange attractors. These attractors play the role of institutional forms satisfying isolated (democratic) demands. And isolated trajectories (democratic demands) are all alike in the sense that all of them lie in the basin of attraction of an attractor that will, sooner or later, capture them. This 'homogeneous' scenario can be considered the analogue of the institutionalist totality.

\footnotetext{
$54 \quad$ Ibid., p. 79.

55 Ibid., p. 74 .
} 


\section{Populism}

Moving away from this scenario, let us imagine that repellers inaugurate interior frontiers and that the homogeneity mentioned above is broken. Laclau speaks of a fractured space, of a division into camps. But how is this division created? Who creates it? How is space effectively fractured? Does the break admit representation in phase space? If not, how can it be read in terms of the analogy? The borders of the manifold in which trajectories coexisting with a strange attractor move are frontiers between zones of phase space where there are trajectories and zones of space which are empty. However, these empty spaces are not representative of the action that empties them. In the case of a deterministic system, the "power" that "creates" these voids is legislated by the rule governing the system. These voids may be created in phase space by changing the mathematical rule that governs the system. But who produces the changes in the rule? The natural answer is "the system." But the terms "rule" and "system" are often used interchangeably. A distinction can, however, be made between the system generating the data and the rule that models it. The system can unexpectedly exhibit a behaviour that is not prescribed by the rule that was supposed to describe it. This makes the model that is expressed by the rule inadequate and calls for a re-adjustment of the rule. The system therefore exceeds the representation of its behaviour in phase space, and constitutes 'something out there' that may force the modification of the representation that is initially chosen as appropriate. This is what creates the fracture in the original context.

But what about the fracture as a political category? The dynamics of the system has borders delimiting voids in phase space, but if we ask about the action behind the void, our relational playground (phase space) remains voiceless. Let us consider an example: let us imagine that we describe a physical system in which two elastic masses collide periodically against each other under the action of certain forces. Let us suppose that we choose one of these masses and that we represent the velocity and position of this mass in phase space. A collision with the other mass may be modelled using a nonlinear term in the mathematical rule (a saturation term). This nonlinear term produces the effect of stopping the mass that we are looking at (the mass that plays the role of our system). Our mass cannot visit certain areas of phase space due to the action of this saturation term. But this forbidden region in phase space inaugurated by the saturation term in the rule does not tell us if the mass is drastically stopped in its motion at a certain position because it is colliding against another mass, or if 
this periodical stop is caused for another reason. The reason is beyond what can be expressed in our representation frame. In terms of the analogy, we can say that the concrete actions that are responsible for the existence of unsatisfied demands do not constitute part of the partial totality in which the demands move. Emptiness is the only visible effect in the relational playground to which meaning is restricted. This allows us to say, with Laclau, that the fracture we are dealing with is a purely negative object. "The void points to the absent fullness of the community." ${ }^{66}$ The holes or fractures in phase space are also a negative representation of a positive action exerted 'out there'. These actions are represented in phase space as regions expelling trajectories, regions where stability or long-term survival becomes impossible. But if there is objectivity only within a certain representation frame, what happens with objects that are constituted in different frames? Is there a possible encounter between such objects? This point will be taken up when we discuss the category of heterogeneity.

Let us move onto the second precondition for the emergence of populism. The transition towards the populist identity consists in the establishment of an equivalential bond between demands. In the parallel we are developing, this equivalential bond takes place with the establishment of a local equivalence relation that enables the inscription of temporally bounded demands into a cell. As expected, the field of the trajectories that are inscribed into a cell is not a "neutral terrain." The "uneasy alternation between cold and warm" demands in Laclau ${ }^{57}$ is the analogue of the contradictory processes of dispersion and contraction leading to a topological invariance.

The inscription within an equivalential chain "gives the demand a corporeality which it would not otherwise have. It ceases to be a fleeting, transient occurrence, and becomes part of [...] a discursive/institutional ensemble which ensures its long-term survival." ${ }^{58}$ We have seen how this inscription leads to the template, the skeleton articulating originally disperse states. The template expresses the long-term survival of the trajectories inscribed in the complex. This long-term survival is expressed in terms of topological invariance. But the par-

\footnotetext{
56 Ibid.., p. 170.

57 Ibid., p. 89.

58 Ibid.
} 
ticular topology of the template represents something on its own: a particular class of articulation, a particular class of dynamics. ${ }^{59}$

This makes the template hypostatic in the following sense. Once constructed, the template stands by itself and does not coincide with the time series that was used to unveil it. The template expresses a property of the system; it represents what survives in the long term if the system is well described by the data. In a way, the time series data is like Wittgenstein's ladder, which can be dropped after climbing. The template overrides the particularistic trajectories and produces meaning ${ }^{60}$ by itself. This hypostasis realises the third and final precondition for the emergence of populism.

\section{Empty signifier}

Lacan holds that the concepts of signifier and articulation are consubstantial, that all that is presented as articulated is the signifier, and at the same time, that the signifier secretes a remnant which it cannot signify. In this sense, every articulation is a failed articulation. We have already discussed in what way phase space is a failed totality. We have already mentioned that phase space exists when we deliberately choose some variables that are considered significative and that will therefore "construct meaning." ${ }_{61}$ This is in line with the manner in which Laclau defines the term system: a system is a failed totality that constitutes a horizon. ${ }^{62}$ The excluded categories cancel their differences through an equivalence that holds between all that is excluded. The system can signify itself as a totality at the price of obliterating (or putting between brackets) the differential nature of what is left out. But all that is obliterated or ignored at a certain time scale or period may not be insignificant at every time scale or peri-

59 "The 'people' does not act as a clearing-house for the individual demands, for it is transformed in most cases into a hypostasis which starts to have demands of its own." Ibid., p. 89. This meaning is more general than the particularistic trajectories, but it is still particular: it is a particular kind of articulation, with a certain topology.

${ }^{61}$ Phase space is the analogue of the "zero point of signification which is nevertheless the precondition for any signifying process.” Ibid., p. 72.

62 "The systematicity of the system is a direct result of the exclusionary limit, it is only that exclusion that grounds the system as such.” Laclau, Emancipation(s), p. 38.

"Any system of signification is structured around an empty place resulting from the impossibility of producing an object which, none the less, is required by the systematicity of the system.” Laclau, On Populist Reason, p. 40. 
od. If "totality now becomes the name of a horizon and no longer of a ground," 63 phase space is also a horizon rather than a ground and is therefore something of the order of an empty signifier. ${ }^{64}$

Let us focus on the cell complex. In the departure context, the cell complex is a methodological element that is built to unveil a topological structure. The popular identity is the analogue of the template. But to determine the topology of the template, the moment of the cell complex construction is critical. The method depends on a performative criterion; different criteria will produce different templates and the success of the program relies on a good choice of this criterion. We have mentioned that the constitution of cells and equivalential chains relies on certain arbitrary criteria, by virtue of which the range of the equivalential process is decided. If the topology of a cell complex depends on a criterion, the method may lead to false rules. This ambiguity is problematic in the original context (since the rule is the scope of the topological program), but in the context of political theory this ambiguity is desirable. In the topological program, the cell complex is methodological, while in the analogy the complex is a constitutive element, with a role that is determinant for cementing the articulation. In other words, while in the original terrain there is a correct template that the method may or may not adequately capture, in the analogy the template does not pre-exist the complex but is legitimately constituted through it. ${ }^{65}$

The cell complex mediates between the clouds of disperse elements and the possibility of constructing the template. The analogy is better tuned if this mediation is seen as a nominal operation. The name operates as a pure signifier, as a structuring element without proper content. The performative operation by which the cell brings about the unity of disperse elements is done in the name of a criterion which cannot be deduced, it has to be defined. The unity of the template is a retroactive effect of its constitution through the criterion leading to the cell complex. This criterion is a relation between universality and particularity, between a particular content in phase space (points distributed in a certain manner) and the universal function embodied by the criterion. Arbitrariness is involved both when phase space is constructed and in the passage from the dis-

\footnotetext{
63 Ibid., p. 103.

64 Ibid., p. 71.

65 Ibid., p. 68.
} 
perse points to the topology that organises them. The structuring dimension (of signification) and the arbitrary (or affective) dimension ${ }^{66}$ converge in the construction of the template and in the construction of the popular identity.

\section{Antagonism, dislocation, and heterogeneity}

According to Laclau, demands may receive the structural pressure of rival hegemonical projects. In the analogy, this occurs for instance when a bifurcation is created in a template so that a single branch opens up into two branches. Trajectories close to the bifurcation will receive the 'structural pressure' of rival branches.

In the original terrain, topological changes in a structure can originate in three increasingly radical ways: through variation of the parameters of the rule, by a change in the form of the rule, or by a change in the definition of phase space. Dynamicists call events that do not involve a redefinition of phase space perestroikas. Phase space reformulation is necessary if the change is manifested, for instance, through the inclusion of previously excluded variables. This distinction between the different manners that may originate structural instability in a template has an almost direct translation into the Laclausian concepts of antagonism, dislocation, and heterogeneity.

Antagonism and dislocation are perestroikas: they do not involve a reformulation of the discoursive inscription. Frontiers play the role of antagonistic relations, and its possibilities of displacement and modification are affine to dislocation. The concept of social heterogeneity, instead, has a type of "exteriority" with respect to the field of representation that is analogous to the most radical change, implying phase space reformulation. ${ }^{67}$

The notion of exteriority, however, remains problematic. In the paragraph devoted to populism, it was mentioned that frontiers and voids in phase space have an apparential exteriority. An antagonism may induce the bifurcation of a branch into two branches. But the exterior whose correlate is the void between the separated branches operates inside the scheme through the mobility of the frontiers of the topological structure. These voids structuring the template intro-

\footnotetext{
66 Ibid., p. 117.

${ }_{67}$ Ibid., p. 148.
} 
duce an element that functions as the internal negation of the pseudo-totality constituted by phase space. ${ }^{68}$

Immanence and transcendence are interwoven in the portrait developed by the analogy. The void opened by the repeller has an apparent exteriority: it can be seen as the correlate of the "intrusion" of something external that operates in phase space causing changes in the topological structure. ${ }^{69}$ These voids are mobile, since the borders of the branches move to allow for a bifurcation. Laclau uses the category of "floating signifier" to refer to the logic of the displacements of the frontiers. The empty signifier (represented in the analogy by the process allowing for the nucleation of points in cells) takes for granted the presence of a stable frontier, while the floating signifier does not. ${ }^{70}$ The distinction is only analytical, since both empty and floating signifiers are complementary categories that participate in the construction of the popular identity. Empty signifiers are involved in the reference to a partial fixation, while floating signifiers ${ }^{71}$ allow for the mutability of these partial fixations. Similarly, the organisation of holes and borders fix a topological structure, and perestroikas allow for the mutability of this organisation.

\section{Section 4. Conclusions}

Ernesto Laclau has rescued the term populism from its pejorative condition. The theory of dynamical systems and topology are fields that have rescued the value of 'the qualitative', making it compatible with mathematical tools that seemed restricted to quantification. This work restores the value of 'the analogical' to apply tools borrowed from the naturalist paradigm to categories of philosophical order.

68 "The multiple presence of the heterogeneous in the structuration of the popular camp is that the latter has an internal complexity which resists any kind of dialectical homogenization. Heterogeneity inhabits the very heart of a homogeneous space." Ibid., p. 152.

69 This intrusion is the analogue of the "heterogeneous other" that is dialectically irretrievable in the emergence of an antagonistic frontier. Ibid., p. 172.

$70 \quad$ Ibid., p. 133.

${ }_{71}$ "The 'floating' dimension becomes most visible in periods of organic crisis, when the symbolic system needs to be radically recast.” Ibid., p. 132. 
'The political', seen as an undecidable game between the floating and the empty, has an analogue in 'the topological' in the structuration of a dynamics. ${ }^{72}$ The conjunction between topology and dynamics has an exponent in the so-called topological program. This work proposes an analogomorphism between the concepts of the topological program and the categories that Laclau defines to articulate the dissatisfaction of demands and the construction of the popular identity. The term analogomorphism is coined here to refer to an ensemble of relationships between terms of two disparate fields, in which the relationships have a heuristic function, and in which the contextualised role of the terms in the original fields can be denatured or transformed by the "mise en relation." In this analogomorphism, the topological program is invoked as a toolbox in the Foucaultian sense of the term. The contextual displacement is hermeneutical, the key being the identification of a trajectory with a demand, and the identification of a stationary state with the satisfaction of a demand. The analogy is not based on borrowing mathematical inscriptions or symbols, but on the common articulation of an ensemble of concepts. This differs from the usage of mathematics implemented by other authors, such as Jacques Lacan or Alain Badiou.

In the original context, the mathematical tools of the topological program are used to construct a skeleton that articulates trajectories in phase space. Here it is argued that the concept of phase space involves the category of hegemony, and that the topological skeleton can be related to the category of "people."

The template has a structure that is topological in nature; it is defined in terms of the number of holes, enclosed cavities, branches, etc. Substantial (qualitative) changes in the system's behaviour affect the topology of the template: enclosed cavities are modified, branches bifurcate, holes appear or close, etc. The concepts involved in the program can be divided into three groups:

- those involved in the representation of the dispersion of elements available before the construction of the template;

- those concerned with the manner in which the template is constructed from the dispersion of elements;

$72 \quad$ Ibid., p. 153.

73 The connections between the terms need not be exhaustive or bijective. 
- those necessary to describe the different possibilities regarding the mutation of the topology of the template.

The concepts in the first group admit a correspondence with the categories of discourse, demand, difference, and institutionalism. Those in the second group are related to the categories of frontier, empty signifier, equivalence, and populism. The concepts in the third group function as analogues for antagonism, dislocation, and heterogeneity.

The template, constructed over a series of methodological steps, is the analogue of the popular camp. Its corporeality is translated in terms of topological invariance. The methodological steps play a central role in the analogy. The concept of template in the analogomorphism is denatured in that it no longer condenses a dynamics that must be unveiled, but a dynamics that is built provided the inscription criteria that cement the articulation operate conveniently. The arbitrariness of these criteria is also essential to the analogy. We could assert that there is a constitutive link between the template-people and the trajectories-demands that articulate it. This link expresses both its strengths and weaknesses. The template is a partial fixation, an open invariant that can be subverted, with subversion conditions that take the form of an alteration of frontiers or of a radical reformulation of the discursive space.

The analogomorphism can be used as a guide to gain insight into several questions that are not discussed in this article. One of them is the distinction between equivalence and homology, i.e. between the equivalential bond at the level of the cell and the equivalential bonds at the level of the complex, in terms of political theory.

Naturally, the type of analogical work proposed in this article need not be restricted to the topological program. Nothing prevents invoking other concepts of the naturalist paradigm with a similar strategy. For instance, the concept of coherence, used in physics to denote certain correlation properties between magnitudes (waves, atoms, magnetic dipoles, etc.), could be proposed, in principle, as a concept with some relationship to the category of hegemony. However, when a physical system attains a coherent state of some kind, the order is directly introduced at the level of the differences. A collective action achieved by a set of individuals could be read as a coherent action in the physical sense of the term, but 
in this case the coherence, the convergence in the arrangement, the order in the action, is attained by cancelling differences. The equivalential logic that builds the topological structure expressed in the template is an order of a different kind. Order and disorder coexist without disturbing each other. Moreover, not only do they coexist peacefully, but they support each other. Let us remark that if we force an order upon the trajectories-demands, disorder disappears and we fall back into the institutionalist limit: trajectories become stationary and demands encounter satisfaction. The analogy with the template is appropriate because the template is not a physical force that orders trajectories into a structure, but rather a sort of containment frame. The template hosts trajectories without dissolving their differences. Differences are sheltered by virtue of an arbitrary criterion that we have related to the affective dimension. Topological invariance, the "ordre dans le chaos," is a product of the coexistence of non-coincident levels: the level of the trajectories or demands, and the level of the template or the people. At the level of the trajectories, differences and disorder persist. The dynamical order that is expressed by the template is not directly or ostensibly present at the level of the trajectories and in this sense it is more discursive than phenomenological, as much as the template is 'more mathematical' than 'physical'. The notion of coherence is therefore more akin to the notion of a general consent, while the notion of equivalence in the topological program allows for an order without convergence, without consensus, without coincidentia. ${ }^{74}$

'The popular', 'the qualitative', and the 'analogical' have a pejorative charge that this text has attempted to overcome by showing the fertility of an exercise that is not limited to a mere parallelism, since it opens up a transfer process of notions, methods, and images. The procedure naturally raises the question of the justification of such an affinity. It also highlights the importance of not reducing an analogomorphism to an isomorphism..$^{75}$ Every analogy is ultimately dialogical and the construction of the analogy unfolded in this work implies a pre- and re-interpretative work, both of the dynamico-topological concepts as well as of Laclausian categories. Analogomorphisms can be put to work in

74 Perhaps the difficulty in harmonising categories such as multitude in Toni Negri and hegemony in Ernesto Laclau can be traced back to the difference between the concepts of coherence and equivalence, in physics and mathematics, respectively.

75 Some of the flaws of Alain Badiou's effort to build an ontology inspired by set theory could be the result of forcing an isomorphism there where an analagomorphism would be more natural. 
political theory to complexify certain political notions, to pose some theoretical questions diversely, or to increase the precision of the manner in which alternative theories proceed. On the other hand, mathematical or physical concepts can profit from analogomorphisms in a genre that can be seen as a deconstructive reflection of scientific practices that are often engaged without consideration of the latent notions with which they operate, those notions that Henri Poincaré once termed mathematical prejudices.

\section{References}

Badiou. Alain, The Concept of Model: An Introduction to the Materialist Epistemology of Mathematics, ed. and trans. Zachary Fraser and Tzuchien Tho, re.press, Melbourne 2007

Bergé, Pierre, Yves Pomeau and Moniques Dubois-Gance, Des rythmes au chaos, Editions Odile Jacob, Paris 1994

Bergé, Pierre, Yves Pomeau and Christian Vidal, L'ordre dans le chaos: vers une approche déterministe de la turbulence, Hermann, Paris 1988

Caveing, Maurice and Évetyne Barbin, “Les Philosophes et les Mathématiques”, Raison présente 123 (1997)

Herrera, Amilcar O., et al. ¿Catástrofe o nueva sociedad? Modelo Mundial Latinoamericano. Centro Internacional de investigaciones para el Desarrollo, Agencia Nacional de Investigación y Desarrollo, Santiago de Chile 2004

Gilmore, Robert and Marc Lefranc, The Topology of Chaos: Alice in Stretch and Squeezeland, Wiley, New York 2002

Laclau Ernesto, Emancipation(s), Verso, London and New York, 2007

- On Populist Reason, Verso, London and New York 2005

Laclau Ernesto and Chantal Mouffe, Hegemony and Socialist Strategy, Second Edition, Verso, London 2001

Ladrière, Jean, El reto de la racionalidad. La ciencia y la tecnología frente a las culturas, Ediciones Sígueme, Salamanca/UNESCO, Paris 1978

Umberto Eco a Pitagora, Le interviste impossibili, Bompiani 1975, available at: http:// www.giutor.com/doc/pitagora/eco-pita.html

Petitot-Cocorda, Jean, Morphogenèse du Sens, PUF, Paris 1985

Sciamarella, Denisse and G. B. Mindlin, “Topological Structure of Chaotic Flows from Human Speech Data”, Physical Review Letters 82 (1999), No. 7

- "Unveiling the Topological Structure of Chaotic Flows from Data”, Physical Review E 64 (2001), No. 3

Tasić, Vladimir, Mathematics and the Roots of Postmodern Thought, Oxford University Press, Oxford 2001

Žižek, Slavoj, The Sublime Object of Ideology, Verso, London and New York 1989 